\title{
El saber escolar en biodiversidad en clave para resignificar su enseñanza
}

Lizeth Paola De la Cruz González ${ }^{1{ }^{\circledR}}$, Nabi Del Socorro Pérez Vásquez ${ }_{\text {Universidad de Córdoba-Colombia }}^{\circledR}$

Autor de correspondencia: 1delacruzgonzalez@correo. unicordoba.edu.co Recibido: 06 de junio de 2020 Revisado: 05 agosto de 2020 Aprobado: 05 de septiembre de 2020 Publicado: 16 de noviembre de 2020

\section{Resumen}

Se presenta una síntesis de resultados de una evaluación formativa realizada a estudiantes de básica secundaria. El propósito fue indagar sus concepciones de biodiversidad para develar las necesidades formativas que requieren fortalecerse en la enseñanza de este campo de conocimiento. Se siguió una metodología cualitativa y, desde el paradigma hermenéutico, se emplearon técnicas como entrevistas semiestructuradas, el análisis de organizadores gráficos y la guía de observación. Los resultados evidencian que la mayoría de los estudiantes muestra dominio básico de los contenidos relacionados con la biodiversidad, limitado a los organismos animales y vegetales. Esto refleja un bajo nivel al establecer relaciones, pues se desestiman conceptos como diversidad genética y ecosistémica, adaptación, evolución, entre otros. Así, se requiere fortalecer en los estudiantes el conocimiento relacional complejo a través de estrategias didácticas que resignifiquen el proceso de enseñanzaaprendizaje.

Palabras clave: biodiversidad, concepciones, saberes, enseñanza 


\title{
The school knowledge in biodiversity in terms of re-signifying its teaching
}

\begin{abstract}
A synthesis of the results of a formative assessment conducted with students of junior high school is presented. The objective was to investigate their conceptions about biodiversity in order to reveal the formative needs that require strengthening in the teaching of this field of knowledge. A qualitative methodology was used and, from the hermeneutic paradigm, techniques such as semi-structured interviews, analysis of graphic organizers and observation guide were employed. The results prove that most students have basic competence in the contents related to biodiversity, restricted to the animal and vegetal organisms. This reflects a low level in establishing connections, since concepts such as genetic and ecosystemic diversity, adaptation, evolution, among others, are dismissed. Thus, it is necessary to strengthen in the students the complex relational knowledge through didactic strategies to resignify the teaching-learning process.
\end{abstract}

Keywords: biodiversity, concepts, knowledge, teaching

\section{O saber escolar sobre biodiversidade como chave para re-significar o seu ensino}

\section{Resumo}

Apresenta-se uma síntese dos resultados de uma avaliação formativa conduzida com estudantes de ensino médio. O objetivo foi investigar as suas concepções sobre biodiversidade a fim de revelar as necessidades de formação que requerem um reforço no ensino deste campo do conhecimento. Seguiu-se uma metodologia qualitativa e, a partir do paradigma hermenêutico, utilizaram-se técnicas como as entrevistas semiestruturadas, a análise dos organizadores gráficos e a guia de observação. Os resultados revelam que a maioria dos estudantes mostra um domínio básico dos conteúdos relacionados com a biodiversidade, limitado aos organismos animais e vegetais. Esta situação é um reflexo de um baixo nível no estabelecimento de relações, dado que conceitos como diversidade genética e de ecossistemas, adaptação, evolução, entre outros, são descartados. Por conseguinte, é necessário reforçar nos estudantes o complexo conhecimento relacional através de estratégias didáticas capazes de resignificar o processo de ensino-aprendizagem.

Palavras-chave: biodiversidade, concepções, saberes, ensino

La revisión y la caracterización de las concepciones de biodiversidad se asocian a su emergencia en relación con aspectos biológicos. Este es un concepto de múltiples interpretaciones de tipo biológico, cultural, político, sistémico, epistemológico, filosófico y ambiental, que "entran en contradicción con el saber científico generalmente transferido a diferentes contextos culturales sin cambio en sus definiciones, de manera aséptica, sin valor y sin ideología, con marginación de otras visiones de mundo" (Pérez, 2013, p. 142). 
Las prácticas de enseñanza de la biodiversidad en el contexto escolar aún están limitadas a la transmisión de contenidos programáticos, donde solo se menciona la biodiversidad, las especies y los ecosistemas como conceptos más, de manera aislada. Se desconoce que hacen parte del contexto, por lo que se convierte en un tema de escaso interés o motivación para los estudiantes (García \& Martínez, 2010). Esto los lleva a interiorizar aspectos básicos que no generan movilidad de los saberes. Como lo menciona Perrenoud (2008), la escuela ha deseado siempre que los aprendizajes que proporciona sean útiles para desarrollar competencias al unir los saberes y su puesta en práctica en situaciones complejas, pero continuamente pierde de vista esta ambición global.

En relación con el concepto aislado o escaso de biodiversidad, se han realizado algunos estudios que afirman que la temática suele aparecer de forma incompleta y poco precisa en los libros de texto. No se aborda desde una perspectiva integral, que incluya las dimensiones: sistémica, sistemática, ecológica, ambiental, evolutiva, ética y socioeconómica. Predomina el carácter sistemático basado en la clasificación taxonómica de animales y plantas (Bermúdez \& Longhi, 2015; Bermúdez et al., 2014; Montero et al., 2013; Valladares, 2011). Otros estudios demuestran que existen debilidades de aprendizaje que incluyen la falta de conocimiento en torno a la biodiversidad y a sus niveles de complejidad - especie, ecosistémica y genética(Guarnizo et al., 2015), por lo cual es importante abordar su tratamiento didáctico y tener en cuenta la identificación de problemáticas emergentes como concepto articulador del desarrollo de las competencias científicas, desde la doble perspectiva de la alfabetización científica y la educación ambiental (Fuentes, 2015; García \& Martínez, 2010; Herrera, 2011; Palacio, 2014).

Se destaca que la educación tiene el reto de la formación integral, apoyado en el desarrollo de habilidades, destrezas, capacidades y saberes integrados en los estudiantes que los lleven a ser críticos, reflexivos y analíticos, con conocimientos sistémicos e integrales que abarquen ejes sociales, ambientales, científicos, académicos, técnicos y tecnológicos; capaces de formular y plantear hipótesis, dar respuesta a situaciones del contexto social y ambiental, para proyectarse como sujetos propositivos y conscientes de su entorno. Estas exigencias convocan al profesorado a proponer modelos que impliquen modificaciones profundas, no solo de carácter curricular, sino en todo el engranaje de los procesos de enseñanza.

De acuerdo con Shulman (1986), el docente debe contar con tres tipos de conocimientos en relación con la práctica educativa: conocimiento del currículo, conocimiento de la materia a enseñar y conocimiento pedagógico. Desde el conocimiento del currículo, son importantes las diferentes significaciones, teniendo en cuenta aspectos relacionados con hechos, conceptos, principios, modelos y teorías, lo cual define los procesos, técnicas y metodologías utilizadas en el campo de aplicación de la biología por el ejercicio docente (Bermúdez \& Longhi, 2012a).

En la actualidad, "se considera que no es posible estudiar un fenómeno relacionado con el conocimiento sin recurrir a la noción de representación" (Tamayo, 2006, p. 41). Esta es importante para aprendizajes significativos, ya que lleva el proceso de enseñanza a salirse de los parámetros tradicionales de interpretación de textos y gráficas y a dar importancia a los procesos de producción y transformación de las representaciones, para lograr el aprendizaje de nuevos conceptos. 
A partir de la perspectiva cognitiva, "las representaciones son consideradas como cualquier noción, signo o conjunto de símbolos que significan algo del mundo exterior o de nuestro mundo interior" (Tamayo, 2006, p. 39). Estas se pueden considerar estrategias de elaboración relacionadas con las técnicas, métodos y formas de representación de datos que permiten establecer conexiones externas e internas entre los conocimientos ya adquiridos por el estudiante, los nuevos contenidos y las formas de organización a través de "agrupamiento, ordenación y categorización de datos, que le permiten al sujeto realizar una representación de la información o contenidos impuestos en clase" (Dorado et al., 2020, p. 79).

Así, los mapas, los dibujos y los diagramas presentados por los estudiantes en torno a sus saberes de biodiversidad, a sus concepciones y a los problemas asociados a ella son representaciones externas, "elaboradas con propósitos comunicativos y producidos por acciones intencionadas denominadas representaciones semióticas, mientras que las representaciones internas aluden a conceptos, nociones y creencias" (Tamayo, 2006, p. 40), las cuales se hicieron evidentes en las entrevistas, a partir de la construcción de marcos interpretativos de los sujetos participantes (Benjumea \& Vélez, 2019). Como no experimentamos la realidad en su totalidad, confiamos en el papel mediador de las percepciones a partir de su reflexión e interpretación, incluso en un nivel básico de cognición. Los signos y su interpretación juegan un papel fundamental para reconocer los saberes sobre un determinado tema (Vogt, 2011).

La enseñanza se relaciona con las estrategias que el docente propone en el aula para que los estudiantes adquieran determinados aprendizajes a través de la mediación, la organización del aula y el uso de recursos didácticos (Fortea, 2019). Para el caso del aprendizaje del campo biológico, el estudiante debe manejar y comunicar lo aprendido mediante gráficos o esquemas que permitan su interpretación, lo cual está ligado al desarrollo de funciones cognitivas relacionadas con la semiosis, en tanto el proceso o acto comunicativo es omnipresente, en este caso, mediante distintos recursos gráficos (Artola et al., 2016). Es importante destacar que "la labor del estudiante tampoco será repetir o recitar lo explicado por el profesor, sino argumentarlo, redescribirlo en función de sus propias teorías implícitas que, con ello, se irán también redescribiendo, explicitando y reestructurando" (Pozo, 1999, p. 518).

Además, es indispensable que la práctica pedagógica y didáctica de los docentes —en el aula y fuera de ella- propicie la evidencia del "saber hacer" del sujeto con el conocimiento adquirido. Es aquí donde la enseñanza contextual es un factor clave del proceso de enseñanza-aprendizaje. Así, surge la necesidad de construir mediaciones didácticas que fomenten la apropiación de la biodiversidad como elemento significativo. Esto permite a los estudiantes situarse en el entorno como parte de ella, como sujetos que asumen la protección de los ecosistemas como espacios vitales para la preservación de la vida y como escenarios de desarrollo personal y social que invitan a la sostenibilidad a través del abordaje de la biodiversidad.

El término biodiversidad fue acuñado por Wilson (1988), quien integró la diversidad biológica en un solo constructo aceptado científicamente como "todas las formas de vida manifestadas en la Tierra, que incluye desde la variación genética de individuos y poblaciones, 
hasta la diversidad de ecosistemas y biomas" (p. 521). Por su parte, la Convención sobre Diversidad Biológica - Convention on Biological Diversity - (2001) define biodiversidad como el conjunto de organismos terrestres y acuáticos. Incluye animales, plantas y microbios en todas las escalas, desde la diversidad genética dentro de las poblaciones, las especies y comunidades a lo largo de los paisajes. Además, la biodiversidad sustenta todos los procesos vitales y contribuye al bienestar humano en la producción de alimentos, de combustible, de fibras y de material genético. Especialmente, proporciona oportunidades recreativas, intelectuales, educativas (Bermúdez et al., 2017).

La biodiversidad comprende todas las formas de vida sobre la Tierra. Se consideran diferentes escalas - temporales y espaciales-, su variedad, su heterogeneidad y las formas de organización de las que son parte (Bermúdez \& Longhi, 2015). En esta investigación la biodiversidad incluye todas las formas de vida agrupadas en los reinos de la naturaleza que viven en un espacio determinado. Además, se destacan la variabilidad genética incluso dentro de los organismos de una misma especie-, los diferentes ecosistemas y los hábitats del planeta con sus particulares condiciones climáticas, además de la variedad de adaptaciones de los organismos y las relaciones e interacciones que se presentan entre seres vivos y el ambiente. El artículo muestra los hallazgos investigativos de un trabajo de maestría con el propósito de indagar los saberes y percepciones de los estudiantes sobre biodiversidad, como fundamento para la elaboración de mediaciones didácticas enfocadas en su reconocimiento.

\section{Metodología}

La investigación se ubicó en el enfoque cualitativo, orientado a ampliar el conocimiento de los fenómenos y a promover oportunidades para tomar decisiones informadas en la acción social (Iño, 2018). Se adopta el paradigma de la doble hermenéutica de Giddens (1984) apoyado en una crítica interpretativa de hechos ya interpretados. De acuerdo con este autor, "el mundo social significativo está constituido por los actores y los metalenguajes inventados por los científicos sociales, en constante deslizamiento de uno al otro inmiscuido en la práctica de las ciencias sociales" (p. 374). Esta metodología hace uso de la comprensión de lo descrito y explicitado por los estudiantes a partir de un ejercicio interpretativo intencional, explicativo y contextual. Se desarrolla la inteligibilidad del discurso contenido en el texto de la representación de la biodiversidad, así como en las voces de los alumnos, al traspasar límites contenidos en sus sentires expresados a través de sus palabras para lograr la captación del sentido. Asi, el análisis hermenéutico en permanente apertura es producto de la actividad reinterpretativa que el autor hace del fenómeno determinado.

El estudio se llevó a cabo con estudiantes de grado $9^{\circ}$ en una institución educativa privada de Montería, Córdoba -Colombia-, la cual cuenta con áreas y zonas verdes propicias para intervenciones didácticas que aprovechen la diversidad presente, desde situaciones contextuales que posibiliten la apropiación de la realidad de los estudiantes sobre la naturaleza circundante, a través de datos sistemáticos para el posterior análisis descriptivo e interpretativo.

Desde las acciones metodológicas, se aplicó un instrumento diagnóstico sobre biodiversidad. Además, para el registro de los saberes se hizo una entrevista a grupos focales. Igualmente, se usó la técnica de recolección de información a través de los organizadores 
gráficos, uno de los mejores métodos para desarrollar las habilidades de pensamiento. Las técnicas de organización gráfica de ideas enseñan a los estudiantes a clarificar su pensamiento y a procesar, organizar y priorizar nueva información (Arévalo, 2015).

Los resultados de las entrevistas a los grupos focales se analizaron en el programa Atlas ti versión 7. Para fortalecer la coherencia y el rigor de los procedimientos analíticos de la investigación, se realizó la sistematización y categorización de los datos y la codificación y agrupación de los códigos y las categorías - familias-. Se obtuvieron las redes semánticas [networks] utilizadas para representar y plasmar el análisis de los datos al mostrar las asociaciones de los saberes de los estudiantes (Hernández et al., 2006).

\section{Resultados}

\section{Saberes de los estudiantes sobre biodiversidad}

Se identificaron los saberes de los estudiantes, apoyados en una interpretación hermenéutica, al reconocer sus representaciones, sus sentires, sus miradas y sus creencias. En este sentido, el saber es la creencia más razonable de todas, por ello no hay entre creencia y saber una diferenciación precisa.

Así, Perrenoud (2012) reconoce que en las acciones humanas son importantes los conocimientos. Aunque no son los únicos recursos movilizados, son determinantes en la acción racional. Nadie actúa sobre la realidad sin hacerse de ella un modelo. Esto va más allá de una simple imagen mental del objeto de la acción, lo cual constituye una especie de teoría de causalidades en acción (Perrenoud, 2012). En el trabajo investigativo, los conocimientos se relacionan como una aproximación a un sistema de saberes y prácticas que esbozan elementos de relación, comprensión y proyección (Pérez, 2019). La representación se entiende como "una forma mental del conocimiento, en la cual quedan abstraídas las cosas del mundo físico y simbólico, bajo la forma de representación" (Esparza \& Rubio, 2016, p. 9). De esta forma, se reconoce al ser humano como entidad simbólica, es decir, que hace uso de la representación mediante la imaginación y la abstracción, al valerse del análisis, de la síntesis y de la creatividad.

Teniendo en cuenta las representaciones gráficas realizadas por los estudiantes, se agruparon las concepciones plasmadas en estas y posteriormente se categorizaron los tipos de saberes según los elementos representados, la simplicidad o complejidad mostrada en la abstracción del concepto de biodiversidad y las problemáticas asociadas a ella. Los saberes se tipificaron como se muestra en la tabla 1.

A partir del análisis de los organizadores gráficos, se evidenció que la mayoría de los estudiantes muestran en sus representaciones un saber básico, al concebir la biodiversidad como un conjunto de organismos, seres vivos, individuos o formas de vida, así como, la variedad de hábitats, espacios o lugares determinados donde se desarrollan estos organismos. Los estudiantes tienen un concepto centrado en la diversidad de animales o plantas. Excluyen los seres vivos pertenecientes a los otros reinos de la naturaleza (figura 1), lo que puede estar relacionado con una abstracción a la etimología de la palabra biodiversidad. Esto los lleva a definirla como "diversidad de vida". De igual forma, se puede atribuir esta apropiación básica del concepto al enfoque sistemático abordado en los libros de texto, que es netamente teórico y centrado en los dos grandes reinos animal y vegetal (Alonso \& Penella, 2013). 
Tabla 1

Tipologías de saberes sobre la construcción del concepto de biodiversidad

\begin{tabular}{|c|c|c|}
\hline CATEGORÍA & $\begin{array}{c}\text { SUBCATEGORÍA } \\
\text { TIPO DE SABERES }\end{array}$ & PERCEPCIÓN \\
\hline \multirow{8}{*}{  } & DIFUSO & $\begin{array}{l}\text { Define biodiversidad como una ciencia que estudia la vida } \\
\text { y aloja todo ser vivo. No se evidencia distinción entre los } \\
\text { reinos de la naturaleza. Se omiten los microorganismos como } \\
\text { bacterias y protistas. }\end{array}$ \\
\hline & BÁSICO & $\begin{array}{l}\text { Define biodiversidad mostrando algunos elementos que } \\
\text { aducen a la variedad de seres vivos, espacios o hábitats, } \\
\text { incluyendo animales, plantas y hongos. }\end{array}$ \\
\hline & & No incluye todos los reinos de la naturaleza. \\
\hline & $\begin{array}{l}\text { RELACIONAL } \\
\text { COMPLEJO }\end{array}$ & $\begin{array}{l}\text { Define biodiversidad haciendo uso de elementos como la } \\
\text { variedad de formas de vida, la variabilidad genética inter- e } \\
\text { intraespecífica, los ecosistemas y las diferentes relaciones } \\
\text { o interacciones que se presentan entre los seres vivos y su } \\
\text { entorno. }\end{array}$ \\
\hline & & $\begin{array}{l}\text { La diversidad de formas de vida abarcando los reinos de la } \\
\text { naturaleza. }\end{array}$ \\
\hline & CULTURAL & $\begin{array}{l}\text { Define la biodiversidad como parte de la cultura enfatizando } \\
\text { en la influencia de la humanidad y sus actividades sobre el } \\
\text { desarrollo de la naturaleza y los elementos que la conforman. }\end{array}$ \\
\hline & & $\begin{array}{l}\text { Algunos estudiantes incluyen la diversidad de religiones, } \\
\text { creencias, costumbres y culturas, grupos indígenas. }\end{array}$ \\
\hline & CONTEXTUAL & $\begin{array}{l}\text { Define biodiversidad enmarcada en el reconocimiento de } \\
\text { Colombia como un país megadiverso con su variedad de } \\
\text { especies, ecosistemas y climas, centradas en la diversidad } \\
\text { regional y local. }\end{array}$ \\
\hline
\end{tabular}

\section{Figura 1}

Representaciones del saber básico de los estudiantes sobre biodiversidad
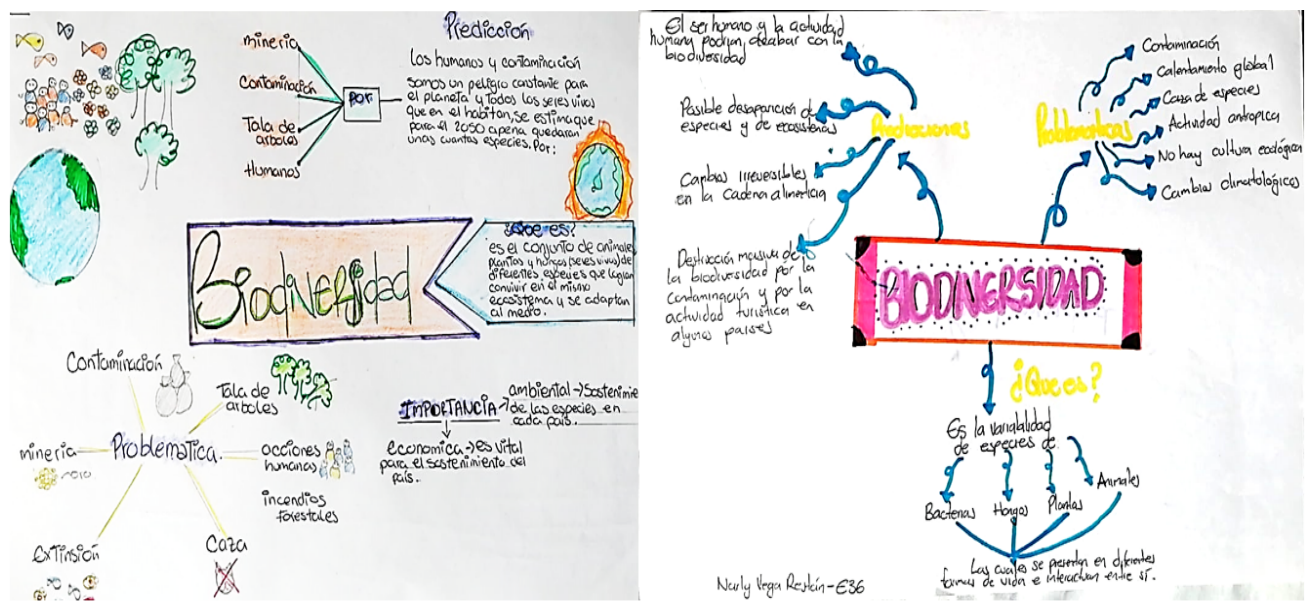

En este orden, se destaca el saber relacional, aquel donde los estudiantes muestran un dominio y comprensión más profunda y estructurada del concepto de biodiversidad, al mostrar en sus representaciones aspectos relacionados con los reinos de la naturaleza, microorganismos - bacterias y protistas_, hongos, animales y plantas, la variabilidad 
genética entre los individuos de igual o diferente población -inter- e intraespecífica一, los diversos ecosistemas y ambientes, incluyendo factores climáticos y su influencia en los organismos y las diferentes relaciones e interacciones que se establecen entre los organismos y el medio (figura 2). Predomina una perspectiva compleja, integral y sistémica, en tanto abarcan los niveles de organización y las dimensiones sistémica, sistemática, ecológica, evolutiva y ambiental de la biodiversidad (Valladares, 2011).

\section{Figura 2}

Representaciones del saber complejo de los estudiantes sobre biodiversidad

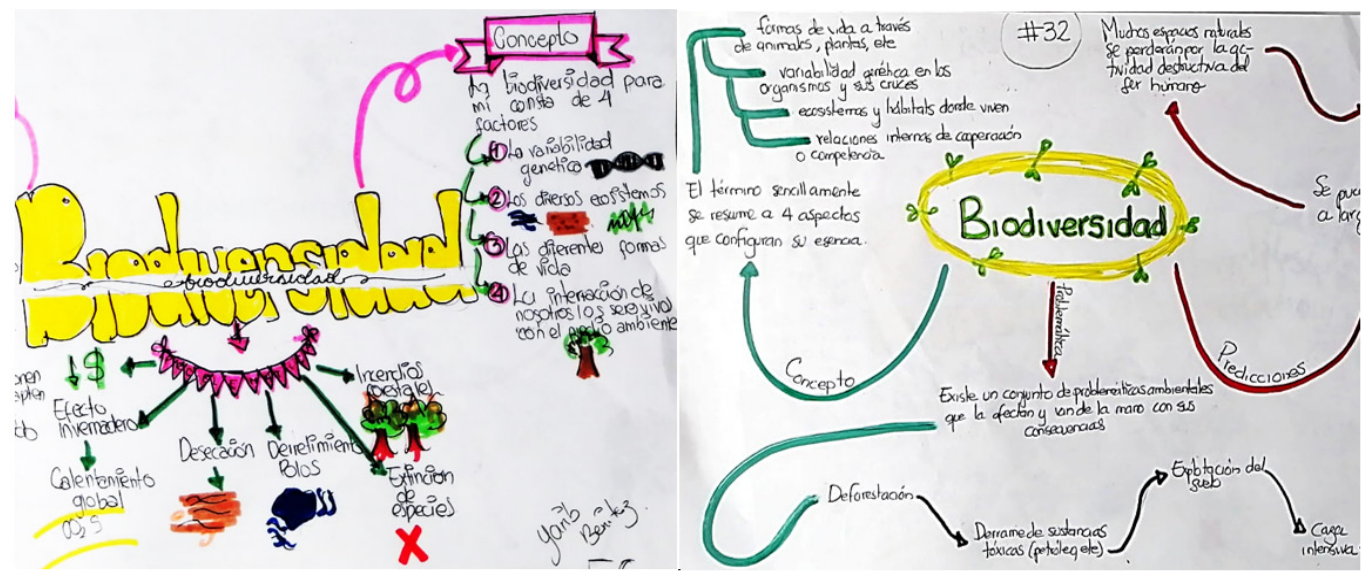

Algunos estudiantes plasmaron ideas y percepciones de la biodiversidad desfasadas, que van desde entenderla como una "ciencia o disciplina que estudia la vida, o los seres vivos", hasta la biodiversidad como el "ente o espacio que aloja todo ser vivo" (figura 3). Estas concepciones fueron categorizadas como saberes desfasados y difusos. Muestran claramente una dificultad en la apropiación, consolidación, dominio y relación conceptual de la temática, lo que puede llevar a que no desarrollen habilidades y actitudes como el respeto y conservación de la misma (Alonso \& Penella, 2013).

Figura 3

Representaciones del saber difuso de los estudiantes de $9^{\circ}$ sobre biodiversidad
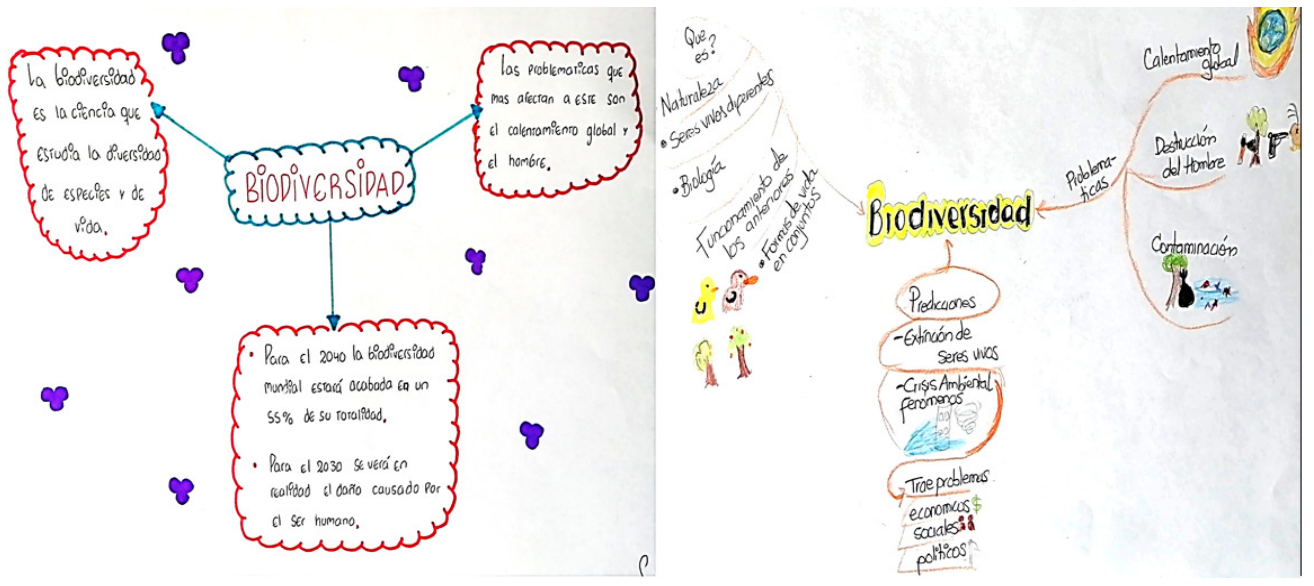

Es importante resaltar que, en el análisis de la información suministrada por las representaciones gráficas de los estudiantes, se evidenciaron definiciones enmarcadas en 
el reconocimiento de la biodiversidad del contexto - saberes contextuales-. Es decir, relacionaban la diversidad como fortaleza de Colombia, desde la riqueza de especies, ecosistemas y climas que lo hacen ubicarse y reconocerse mundialmente como un país megadiverso. Este tipo de saber aparece bajo denominaciones diversas, como son las ideas espontáneas y las creencias que caracterizan el entorno social y cultural del estudiante. Se tiene en cuenta que el conocimiento local o tradicional es parte de los saberes previos de los estudiantes y puede constituir bases para la estructuración de saberes desde una perspectiva constructivista, hasta lograr aprendizajes contextualizados y significativos y un acercamiento a los conocimientos y conceptos científicos propios de las ciencias naturales (Díaz \& Morant, 2017).

El saber contextual se articuló de igual forma con el saber cultural, en la medida en que algunos estudiantes en sus representaciones gráficas plasmaron visiones y creencias en donde relacionan la biodiversidad con la riqueza de culturas, costumbres, grupos indígenas, razas, cultos y religiones, géneros, diversidad de pensamientos y formas de expresión (figura 4). Este saber, centrado mayoritariamente en aspectos sociales y culturales del ser humano, enfatiza en la influencia de la humanidad y sus actividades sobre el desarrollo y equilibrio de la naturaleza y los elementos que la conforman. Dichas visiones son particularmente llamativas, ya que la enseñanza de las ciencias naturales no se debe limitar a conceptos exclusivos de la ciencia, sino que debe permitir la integración de conocimientos que aporten al entendimiento de la complejidad, la recuperación y la conservación de la memoria cultural. A su vez, esto implica una oportunidad de enriquecimiento mutuo, horizontal, complementario y recíproco con las ciencias sociales, para que se pueda construir una propuesta educativa interdisciplinar, inclusiva y alternativa, en pro de la construcción de múltiples miradas de la biodiversidad (Anderson et al., 2015).

\section{Figura 4}

Representaciones del saber contextual y cultural de los estudiantes sobre biodiversidad

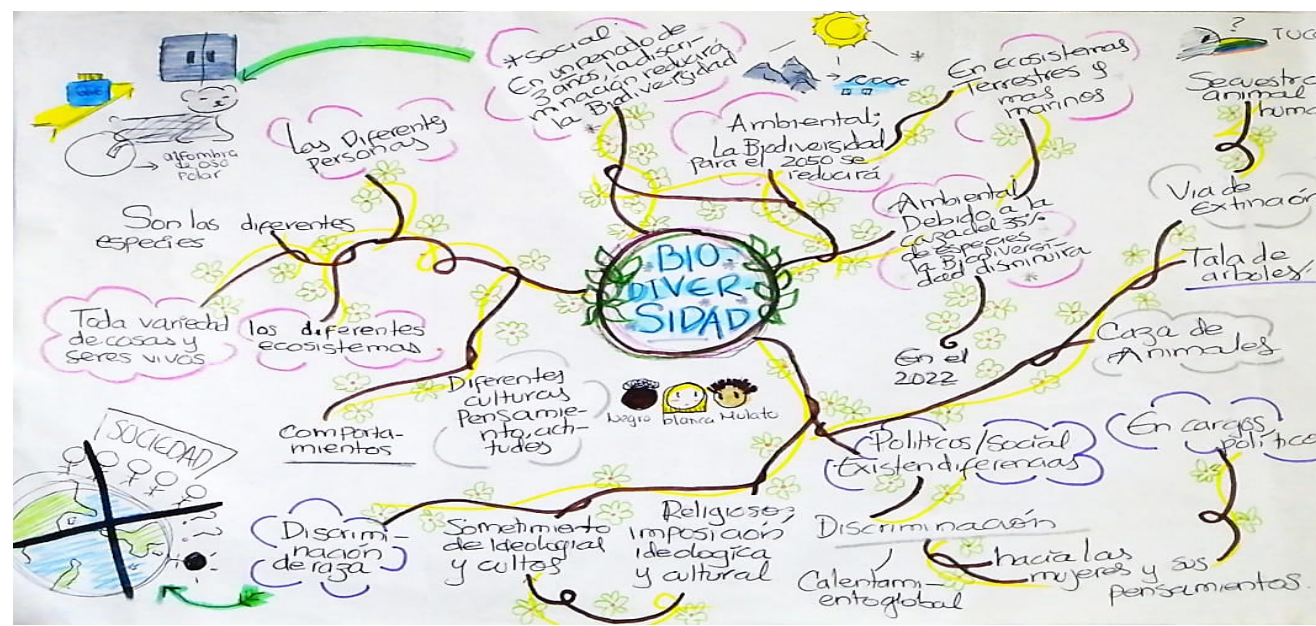

Los resultados de la agrupación y categorización de los saberes sobre biodiversidad de los estudiantes se presentan en la figura 5.

Por otra parte, y con el fin de complementar los hallazgos de los saberes sobre biodiversidad, se realizó un análisis cualitativo e interpretativo de la información. Esto 
permitió la exploración y el estudio profundo de las representaciones y opiniones de los estudiantes, que, además de las representaciones gráficas - mapas mentales_-, abarcaban las expresiones orales y la argumentación. Un análisis de lo que se puede percibir en los gráficos -como emociones, sentires, opiniones, críticas, posturas y actitudes (figura 6) - resulta relevante, ya que se articula con ciertas habilidades de comunicación de las competencias científicas en las ciencias naturales.

\section{Figura 5}

Tipologías del saber sobre biodiversidad

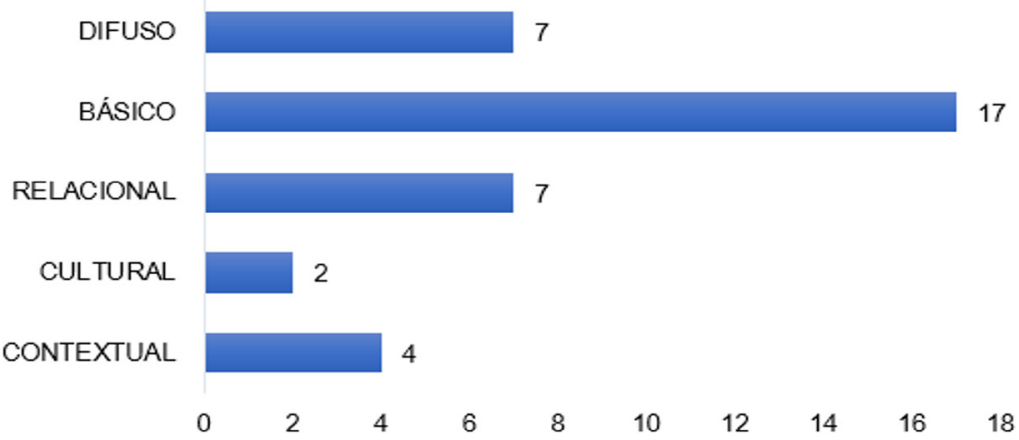

El network evidenció la concordancia entre lo expresado en la entrevista y lo representado en los organizadores gráficos. Así, los estudiantes que cuentan con un saber relacional complejo del concepto de biodiversidad reflejaron dominio conceptual y complejidad en las relaciones al abordar elementos de los reinos de la naturaleza, variabilidad genética, diversidad de ecosistemas y de interacciones de los organismos entre sí y con el entorno. En sus voces:

- E1: la biodiversidad son las diferentes formas de vida que habitan en nuestro planeta ya sea en un mismo o diferentes espacios. Los conceptos para mí son: variabilidad genética, la variedad de ecosistemas, variedad de especies y cómo se relacionan esas especies con el ambiente en el que habitan.

Por su parte, otra estudiante señala:

- E32: el concepto se configura en cuatro aspectos: primero las formas de vida que habitan en estos espacios que se reparten entre seres humanos, animales, plantas, bacterias, etc. El segundo aspecto son las interacciones o las relaciones que existen entre estos organismos. El tercer aspecto es el espacio en que se dan estas interacciones es decir los hábitats. Y finalmente el cuarto aspecto es la variabilidad genética que existe entre ellos.

Esta forma de concebir la biodiversidad permite evidenciar cierto nivel de profundidad en cuanto a la habilidad y capacidad de relación entre conceptos y contenidos que los estudiantes hasta este grado han manejado en su recorrido escolar. Estas acepciones de biodiversidad son realmente complejas, lo cual evidencia la comprensión de contenidos propios de la genética, la ecología y la biogeografía, y devela la complejidad del concepto, que trasciende desde lo biológico-genético hasta lo ecosistémico en las escalas de espacio y tiempo. 


\section{Figura 6}

Network de Atlas Ti donde se muestran los saberes sobre biodiversidad de los estudiantes de grado $9^{\circ}$

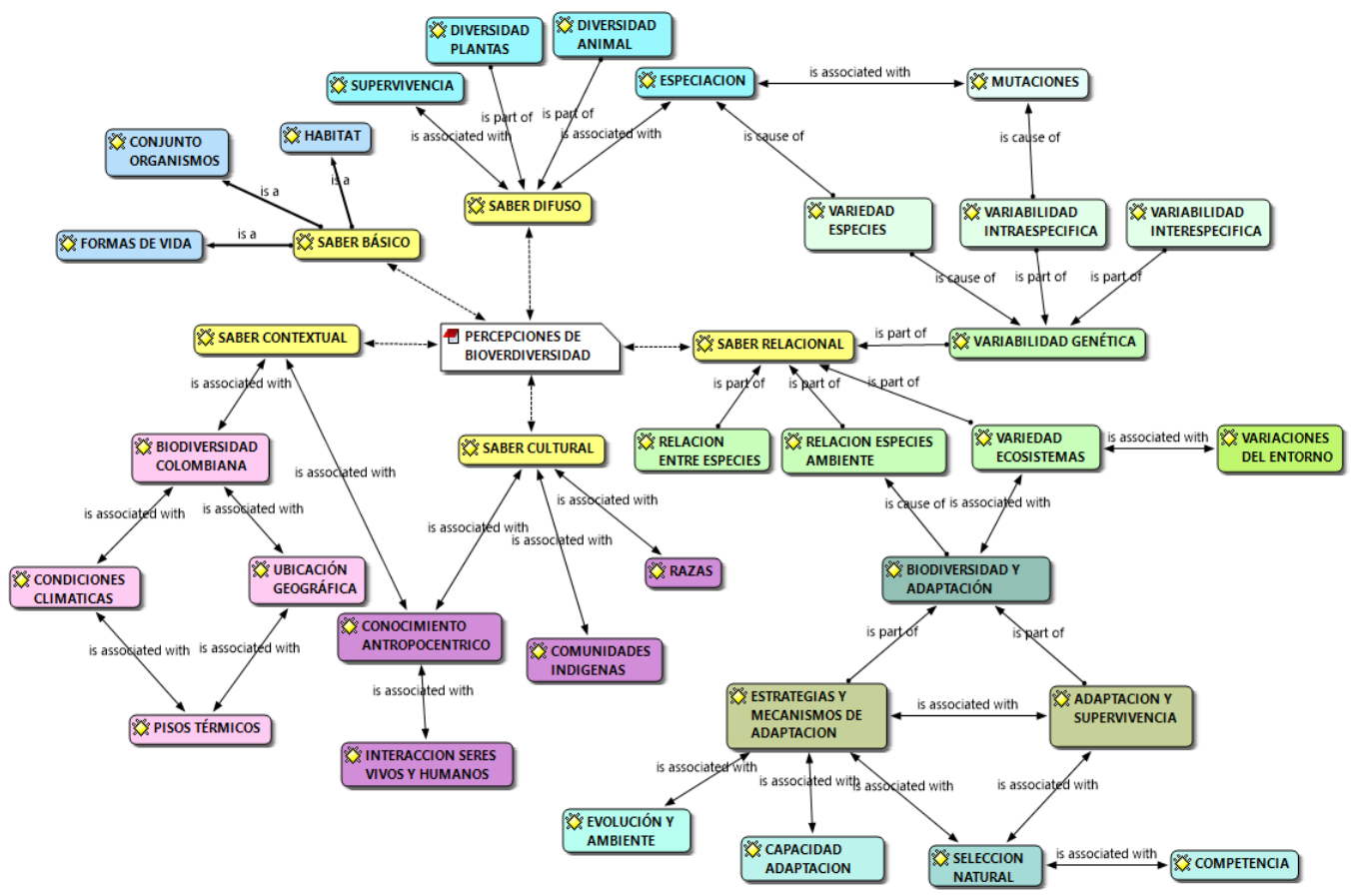

En la figura 6 se vincula el saber relacional a la variedad de conceptos y términos. Llama la atención cómo expresan con gran apropiación contenidos como la variabilidad inter- e intraespecífica, la selección natural y su influencia en la supervivencia y evolución de las especies. Asimismo, demuestran que conocen las estrategias y mecanismos de adaptación que los organismos desarrollan para relacionarse y perpetuarse en medio de un ambiente cambiante que ejerce presiones selectivas sobre estos.

Por otro lado, se destacan en los estudiantes otras perspectivas conceptuales un poco más simples, pero predominantes. Definen la biodiversidad como el conjunto de organismos o diferentes formas de vida que se encuentran en un hábitat o espacio determinado. Otros expresan que corresponde a la diversidad animal o vegetal, lo cual muestra una acepción sistemática de la biodiversidad que impide una visión global y fomenta una visión reducida a los animales y plantas. Esto podría estar relacionado con el aprendizaje en grados escolares menores. La biodiversidad es a menudo entendida solo como la riqueza de especies; es decir, número de especies presentes en un área (Bermúdez et al., 2014). En la medida en que no mencionan la diversidad genética y los ecosistemas, no se evidencia una transposición didáctica que permita una visión holística del concepto de biodiversidad (Alonso \& Penella, 2013). En sus definiciones, los estudiantes reconocen la biodiversidad como:

- E20: conjunto de organismos vivos que habitan en un espacio o en algún lugar.

- E8: es toda la naturaleza, los seres vivos diferentes, el funcionamiento de ellos y las formas de vida en conjunto. Es el conjunto y espacio determinado, al igual que la diversidad de animales y plantas.

Por último, es necesario analizar cómo en algunas de las intervenciones se expresa la diversidad regional de Córdoba, lo cual demuestra que los estudiantes reconocen su contexto, 
aspecto importante, dado que permite el análisis regional, cultural y social asociado a estos saberes, para fomentar el aprendizaje significativo y el cuidado de lo propio. A este tipo de conjeturas las llamamos saber cultural. Las representaciones sociales y el contexto de los estudiantes en su cotidianidad incluyen atributos sociales como la heterogeneidad de razas, clases sociales, creencias, costumbres, religiones y culturas de las civilizaciones humanas. Dichas expresiones no se pueden considerar ilógicas y erróneas, sino más bien integradas, ya que condicionan la enseñanza y/o el aprendizaje de los contenidos, tanto de conceptos, como de los procesos y las actitudes hacia el cuidado de la naturaleza y el respeto hacia la diversidad social (Bermúdez \& Longhi, 2012b). Algunas de las ideas manifestadas por los estudiantes están enmarcadas en estos saberes, pues señalan:

- E20: la biodiversidad en Colombia es muy amplia, muy diversa ya que Colombia es el país más biodiverso por su extensión geográfica, se puede evidenciar en regiones como el Amazonas, Sierra Nevada de Santa Marta y el Chocó. Además, contamos con muchos pisos térmicos y ecosistemas como bosque tropical seco y húmedo.

Otros, por su parte, amplían sus conceptualizaciones al señalar la relación cultural de la biodiversidad y sus ventajas para la conservación:

- E5: nuestro país tiene variedad de razas, como las indígenas y los negros, las culturas, costumbres y tradiciones cambian dependiendo la región, pienso que esto es muy importante en nuestro país que también lo hace ser único y megadiverso.

Según lo anterior, la biodiversidad se configura entonces como un concepto que va más allá de lo biológico y que llega a impregnarse de las experiencias humanas del contacto con las otras formas de vida. Esto refleja una amplia red de interacciones no solo ecológicas, sino culturales y sociales, lo cual genera percepciones, visiones, valores y actitudes que pueden definir comportamientos y prácticas sensibles en la interacción de las comunidades y su ambiente (Álvarez, 2001).

\section{Resignificando la enseñanza de la biodiversidad}

Los resultados relacionados con los saberes de biodiversidad de los estudiantes develan la necesidad de resignificar la enseñanza y el abordaje de esta temática en el contexto escolar, por lo cual se realizan algunas precisiones:

El conocimiento básico mostrado por la mayoría de los estudiantes puede estar relacionado con las estrategias utilizadas por los docentes, en tanto sus desarrollos han sido desde enfoques tradicionales, centrados en la planeación y la puesta en marcha de contenidos. Esto privilegia la acumulación de conocimientos más que el fortalecimiento de habilidades que les permitan a los estudiantes relacionar los componentes de la biodiversidad. La observación de las prácticas pedagógicas generales demuestra que no se desarrollan acciones fuera del aula que permitan integrar el contexto y, en este caso, la biodiversidad que existe en la institución a partir de una comprensión reflexiva, crítica y analítica. Además, en sus apuestas formativas favorecen poco la motivación, la curiosidad, la capacidad de asombro y la investigación, lo que no les permite a los alumnos resolver situaciones problémicas asociadas a la biodiversidad. Se puede afirmar que los procesos formativos desarrollados en el área de ciencias naturales no se articulan al propósito de formar ciudadanos competentes, sobre todo en correspondencia con el campo de conocimientos sobre biodiversidad.

La situación de enseñanza descrita alude a la necesidad del replanteamiento de 
estrategias y métodos de enseñanza como: la experimentación, la observación directa -o mediada por instrumentos-, la modelización y la construcción de narraciones históricas (Castro \& Valbuena, 2018). Estos, sumados a la generación de ambientes de aprendizaje, posibilitarían el acercamiento al pensamiento científico y a los procesos de investigación como el aprendizaje basado en problemas o acciones de aprendizaje basado en proyectos que incluyan la investigación escolar y la indagación como elementos para fortalecer los procesos de aprendizaje en los estudiantes. Lo anterior propiciaría experiencias y situaciones cercanas a sus conocimientos científicos, para, de forma continua, argumentar, primero, desde sus saberes previos y, luego, desde los saberes construidos en relación con la teoría; es decir, argumentar desde la construcción y uso del conocimiento (De la Cruz \& Pérez, 2019), a partir de actividades de investigación o de trabajos por proyectos que generen aprendizaje significativo.

En este contexto, es importante considerar las prácticas de campo como espacios educativos donde los estudiantes participen activamente al relacionar la biodiversidad y cada uno de sus elementos, apoyados en acciones de aprendizaje que ubican el trabajo colectivo en el centro del proceso que lleva a una práctica formativa situada en un contexto de intervención específico. Lo anterior favorece el desarrollo de una cultura ambiental basada en la corresponsabilidad y la participación individual. En este punto, los docentes pueden utilizar estrategias en el fortalecimiento de los saberes en biodiversidad que lleven a los estudiantes a actitudes de respeto y de protección. En cuanto a las estrategias afectivomotivacionales, "generar cogniciones y emociones en los estudiantes con respecto a las actividades de aprendizaje se relaciona con la motivación intrínseca y extrínseca del sujeto" (Dorado et al., 2020, p. 79).

La enseñanza de la biodiversidad puede ir de la mano con el desarrollo de competencias en los estudiantes en relación con las habilidades para reorganizar lo aprendido y transferirlo a nuevas situaciones y contextos: llevar a la práctica lo que se desarrolla en el aula. De acuerdo con Artola et al. (2016), la transferencia del conocimiento no es inmediata; implica previamente la abstracción, lo que requiere dotar al estudiante de las herramientas cognitivas necesarias para reflexionar, controlar y ejecutar mejor su conocimiento - metacognición-.

Finalmente, se propone el uso de enfoques pedagógicos que propicien el trabajo colectivo, la revisión de la situación problemática real, el estudio de caso, proyectos, seminarios problémicos, entre otros, para que haya debate, intercambio y construcción de sentidos, lo cual permitiría la invención, la capacidad de asombro, el desarrollo crítico y las respuestas creativas e innovadoras. Por ello, se propone tomar como punto de partida el contexto de los estudiantes y el abordaje disciplinario de los campos de conocimientos.

\section{Conclusiones}

La mayoría de los estudiantes muestra un dominio básico de los contenidos relacionados con la biodiversidad, limitado a la variedad de organismos animales y vegetales. Se evidencia un bajo nivel de establecimiento de relaciones, ya que se desestiman conceptos como diversidad genética y ecosistémica, adaptación, evolución, ecología poblacional, entre otros, que al integrarse podrían consolidar un saber sistémico y complejo. De igual forma, la inclusión de las problemáticas ambientales y los criterios de tipo social y cultural, al contextualizase a partir de la realidad de los sujetos, pueden condicionar los procesos de 
enseñanza-aprendizaje de contenidos, conceptos, fenómenos y actitudes en los estudiantes. Esto proporcionaría experiencias de aprendizaje significativo, en la medida que conozcan desde las problemáticas hasta las decisiones que deberían tomar al involucrarse con el problema y sentirse parte de él.

Según las representaciones de los estudiantes, se requiere la implementación de estrategias didácticas en la enseñanza de la biodiversidad, que permitan pasar de la frontera de lo biológico hacia la integralidad del conocimiento desde las áreas de ciencias sociales y ética, para ayudar al desarrollo de posturas críticas y reflexivas en torno a la preservación de la biodiversidad y todos sus elementos.

\section{Referencias}

Alonso, C., \& Penella, M. (2013). Análisis del concepto de biodiversidad en los libros de texto de segundo ciclo de primaria en la Comunidad Valenciana (España). Perfiles Educativos, 35(141), 97-114. https://doi.org/10.1016/S0185-2698(13)71837-3

Álvarez, A. (2001). De la herencia cotidiana al tesoro perdido: Nuevos desafíos en la educación ambiental para la conservación de la biodiversidad. Interciencia, 26(10), 429433.

Anderson, C., Pizarro, J., Fontana, R., Sapoznikow, A., Pauchard, A., Barbosa, O., Moreira, A., \& Valenzuela, A. (2015). ¿Estamos avanzando hacía una socio-ecología? Reflexiones sobre la integración de las dimensiones "humanas" en la ecología en el sur de América. Ecología Austral, 25(3), 263-272.

Arévalo, T. (2015). Uso de organizadores gráficos como estrategia de aprendizaje por parte de los estudiantes de sexto grado primaria del Colegio Capouilliez. [Tesis de maestría, Universidad Rafael Landívar]. Repositorio digital Landivariano. http://recursosbiblio. url.edu.gt/tesiseortiz/2015/05/84

Artola, E., Mayoral, L., \& Benarroch, A. (2016). Dificultades de aprendizaje de las representaciones gráficas cartesianas asociadas a biología de poblaciones en estudiantes de educación secundaria. Un estudio semiótico. Revista Eureka, 13(1), 36-52. https:// www.redalyc.org/articulo.oa?id=920/92043276004

Benjumea, C., \& Vélez, J. (2019). Prácticas discursivas y pedagógicas que configuran la identidad Lasallista: una experiencia de aula. Revista Lasallista de Investigación, 16(1), 150-163. https://dx.doi.org/10.22507/rli.v16n1a12

Bermúdez, G., \& Longhi de, A. (2012a). El conocimiento didáctico de contenidos biológicos de Ecología. En C. Flores (Ed.), Experiencias latinoamericanas en educación ambiental. CECyTE NL-CAEIP.

Bermúdez, G., \& Longhi de, A. (2012b). Análisis de la transposición didáctica del concepto de biodiversidad. Orientaciones para su enseñanza. Algunas Problemáticas de Investigación. La Enseñanza de las Ciencias, 32(3), 115-153. https://doi.org/10.5565/rev/ ensciencias. 1129

Bermúdez, G., \& Longhi de, A. (2015). Retos para la enseñanza de la biodiversidad hoy. Aportes para la formación docente ( $1^{\text {a }}$ ed.). Universidad Nacional de Córdoba. 
Bermúdez, G., Battistón, L., García, M., \& Longhi de, A. (2017). Sociocultural Variables That Impact High School Students' Perceptions of Native Fauna: a Study on the Species Component of the Biodiversity Concept. Research in Science Education, (47), 203-235. https://doi.org/10.1007/s11165-015-9496-4

Bermúdez, G., Longhi de, A., Díaz, S., \& Catalán, V. (2014). La transposición del concepto de diversidad biológica. Un estudio sobre los libros de texto de la educación secundaria española. Enseñanza de las ciencias, 32(3), 285-302. http://dx.doi.org/10.5565/rev/ ensciencias. 1129

Castro, J., \& Valbuena, E. (2018). Algunas relaciones entre la autonomía de la biología y la emergencia de su didáctica: consideraciones sobre la complejidad de enseñar una ciencia compleja. Ciência \& Educação, 24(2), 267-282. https://doi.org/10.1590/1516731320180020002

Convention on Biological Diversity. (2001). Sustaining life on Earth. Secretariat of the Convention on Biological Diversity. United Nations Environment Programme. https:// www.cbd.int/doc/publications/cbd-sustain-en.pdf

De la Cruz, L., \& Pérez, N. (2019). Diseño de una unidad didáctica sobre la diversidad de flora para el desarrollo de competencias científicas en el aprendizaje de las ciencias naturales. Bio-grafía, (Extraordinario), 397-406. https://revistas.pedagogica.edu.co/ index.php/bio-grafia/article/view/10870

Díaz, G., \& Morant, A. (2017). Conocimientos sobre biodiversidad en una escuela primaria rural en el sur de Mendoza (Argentina) ¿Diálogo entre educación científica y ambiental? En L. Dubini, M. Erice, D. Luna, M. Astete, \& L. Martínez, (Eds.). Educación científica e inclusión sociodigital: actas del IX Congreso Iberoamericano de Educación Científica $y$ del I Seminario de Inclusión Educativa y Sociodigital (CIEDUC 2017) (pp. 537-544) Universidad de Alcalá, Servicio de Publicaciones.

Dorado, A., Ascuntar, J., Garcés, Y., \& Obando, L. (2020). Programa de estrategias de aprendizaje para estudiantes de una institución educativa. Praxis \& Saber, 11(25), 75-95. https://doi.org/10.19053/22160159.v11.n25.2020.9272

Esparza, R., \& Rubio, J. (2016). La pregunta por el conocimiento. Saber, 28(4), 813-818.

Fortea, M. (2019). Metodologías didácticas para la enseñanza/aprendizaje de competencias. Unitat de Formació i Innovació Educativa de la Universitat Jaume I. http://dx.doi. org/10.6035/MDU1

Fuentes, M. (2015). La diversidad en el marco de la didáctica de las ciencias naturales: una experiencia en el aula de educación secundaria obligatoria. Repositorio Universidade Coruña. http://hdl.handle.net/2183/14786

García, J., \& Martínez, F. (2010). Cómo y qué enseñar de la biodiversidad en la alfabetización científica. Enseñanza de las ciencias, 28(2), 175-184. https://www.raco.cat/index.php / Ensenanza/article/view/199611/353385

Giddens, A. (1984). The Constitution of Society. Outline of the Theory of Structuration. University of California Press. 
Guarnizo, M., Puentes, O., \& Amórtegui, E. (2015). Diseño y aplicación de una unidad didáctica para la enseñanza-aprendizaje del concepto diversidad vegetal en estudiantes de noveno grado de la institución educativa Eugenio Ferro Falla, Campo Alegre, Huila. Tecné, Episteme y Didaxis: TED, (37), 31-49. https://doi.org/10.17227/01213814.37ted25.45

Hernández, R., Fernández-Collado, C., \& Baptista, P. (2006). Metodología de la investigación. McGraw-Hill. https://bit.ly/2ziudpn

Herrera, A. (2011). La conservación de la biodiversidad para grado octavo de la Institución Educativa Distrital los Pinos de la ciudad de Bogotá, una unidad didáctica [Tesis doctoral, Universidad Nacional]. Repositorio Institucional UN. http://www.bdigital.unal.edu. co/8164/

Iño, W. (2018). Investigación educativa desde un enfoque cualitativo: la historia oral como método. Voces de la educación, 3(6), 93-110.

Montero, S., Brown, D., \& García, I. (2013). Evolución del concepto de biodiversidad a través de una secuencia de actividades de ordenación del territorio en un contexto local (presidencia). Enseñanza de las ciencias, (Extra), 2368-2374.

Palacio, V. (2014). Estrategia para la enseñanza del ecosistema de bosque tropical que contribuy a al desarrollo de las competencias científicas en los estudiantes de grado cuarto de la Institución Educativa Federico Carrasquilla [Tesis de maestría, Universidad Nacional de Colombia]. Repositorio Institucional UN. https://repositorio.unal.edu.co /handle / unal/21803

Pérez, M. (2013). Concepciones de biodiversidad: una mirada desde la diversidad cultural. Magis, 6(12), 33-151. https://doi.org/10.11144/Javeriana.m6-12.cbmd

Pérez, M. (2019). Concepciones de biodiversidad y prácticas de cuidado de la vida desde una perspectiva cultural. Tecné, Episteme y Didaxis: TED, (45), 17-34. https://doi. org/10.17227/ted.num45-9830

Perrenoud, P. (2008). Construir las competencias ¿es darle la espalda a los saberes? Docencia universitaria, 6(2), 1-8.

Perrenoud, P. (2012). Cuando la escuela pretende preparar para la vida ¿Desarrollar competencias o enseñar otros saberes? Magisterio.

Pozo, J. (1999). Más allá del cambio conceptual: el aprendizaje de la ciencia como cambio representacional. Enseñanza de las ciencias. 17(3), 513-520

Shulman, L. (1986). Those who understand: Knowledge growth in teaching. Educational Researcher, 15(2), 4-14.

Tamayo, O. (2006). Representaciones semióticas y evolución conceptual en la enseñanza de las ciencias y las matemáticas. Educación y Pedagogía, 28(45), 37-49.

Valladares, L. (2011). Las competencias en la educación científica. Tensiones desde el pragmatismo epistemológico. Perfiles educativos, 33(132), 156-179. http://www.scielo .org.mx/scielo.php?script=sci_arttext\&pid=S0185-26982011000200010\&lng=es\&nrm $=\mathrm{iso}>$. 
Vogt, L. (2011). Signs and terminology: science caught between language and perception. Bionomina, (4), 1-41. https://doi.org/10.11646/bionomina.4.1.1

Wilson, E. (1988). Biodiversity. National Academy Press. 